Classification

Physics Abstracts

$44.50-72.15 \mathrm{c}-81.70$

\title{
Thermal wave characterization of inhomogeneities in sprayed coatings
}

\author{
Ts. Velinov, B. Gergov and K. Bransalov \\ Department of Solid State Physics, Sofia University, 5 blvd Anton Ivanov, 1126 Sofia, Bulgaria
}

(Reçu le 8 janvier 1990, révisé le 2 avril 1990, accepté le 25 avril 1990)

\begin{abstract}
Résumé. - Des couches métallisées par projection thermique ont été examinées par des ondes thermiques et leurs chaleurs spécifiques et conductivités thermiques ont été évaluées. Les déviations des résultats expérimentaux de la théorie des milieux homogènes dans le domaine des basses fréquences servent à déterminer l'influence des différentes espèces de défauts. Les rôles des pores et des barrières thermiques ont été étudiés en comparant les résultats avec des modèles des milieux inhomogènes et on a trouvé que l'influence des barrières thermiques sur les propriétés des couches métallisées par projection est la plus importante.
\end{abstract}

\begin{abstract}
Thermally-sprayed metal coatings were investigated with thermal waves and their specific heats and thermal conductivities were determined. The deviations of the experimental results from the theory of homogeneous solids at low frequencies served to determine the influence of different types of defects. The roles of the pores and the thermal barriers were studied by comparing the results to models of inhomogeneous media and the thermal barriers were found to affect significantly the coating properties.
\end{abstract}

\section{Introduction.}

Photoacoustic (PA) and photothermal (PT) methods are widely applied in the investigation of layered samples and thin films including thermally sprayed coatings [1-3]. The study of the latter is however hampered by their inhomogeneity. The presence of several phases, pores, inclusions, as well as the structural alterations depending on the spraying conditions significantly change the coating properties. On the other hand the specific photoacoustic behaviour may be used to advantage to clarify the contribution of different kinds of inhomogeneities.

In this paper measurements of the thermal conductivity and the specific heat of flame sprayed coatings by means of PA technique are reported and the influence of the deposition conditions on the coating structure and thermophysical properties is examined. The observed deviations from the PA theory of homogeneous solids and the comparison of the experimental data with results from models of inhomogeneous media serve to determine the role of different types of defects in the investigated samples.

\section{Sample preparation.}

The cylindrical specimens with a diameter of $14 \mathrm{~mm}$ were coated by flame spraying with powder onto
$2 \mathrm{~mm}$ thick roughened stainless steel substrates. Some coating parameters are given in table I.

Table I. - Some coating parameters.

\begin{tabular}{|l|l|c|c|c|}
\hline Sample & powder & $\begin{array}{c}\text { thickness } \\
(\mu \mathrm{m})\end{array}$ & $\begin{array}{c}\text { density } \\
\left(\mathrm{g} / \mathrm{cm}^{3}\right)\end{array}$ & $\begin{array}{c}\text { porosity } \\
(\%)\end{array}$ \\
\hline S1 & MOGUL-M48 & $30-35$ & 7.16 & 17.8 \\
S2 & MOGUL-M48 & $55-60$ & 7.16 & 17.8 \\
S3 & MOGUL-M48 & $>1500$ & 7.16 & 17.8 \\
S5 & MOGUL-M48 & $55-60$ & 7.22 & 17.1 \\
S6 & MOGUL-M48 & $>1500$ & 7.22 & 17.1 \\
S9 & MOGUL-M100 & $>1000$ & 6.99 & 25.4 \\
S11 & MOGUL-M135 & 60 & 6.88 & 8.8 \\
S12 & MOGUL-M135 & $>1500$ & 6.88 & 8.8 \\
\hline
\end{tabular}

The technological conditions of preparation of S1S3 and S11, S12 differ from those of the other samples, for example S5 and S6. In the former case air was used to additionally accelerate the projected grains. The particle size was $45-90 \mu \mathrm{m}$ for all samples. The density and the porosity were determined by hydrostatic weighing method and were averaged over several samples. The coating thickness was measured by microscope after cross sectioning the 
samples and greater value was used in the calculations. The powders were produced by Interweld, Austria. The powder MOGUL M-48 represents Ni based alloy with $\mathrm{W}_{2} \mathrm{C}$ and WC additives, MOGUL M-100 is Mo powder and MOGUL M-135 is bronze alloy.

\section{Experiment and discussion.}

The schematic diagram of the experimental setup is shown in figure 1. A Plexiglas cell, designed as a Helmholtz resonator with an about $2 \mathrm{~cm}^{3}$ sample chamber, and a condenser microphone Brüel and Kjaer 4166 were used. The beam of a $50 \mathrm{~mW} \mathrm{He-Ne}$ laser was interrupted by a computer controlled mechanical chopper in the frequency range 13$250 \mathrm{~Hz}$ and fell unfocused onto the sample. The amplitude and the phase of the PA signal were registrated by a PARC 5301 lock - in amplifier and were sent to a computer through a RS $232 \mathrm{C}$ interface.

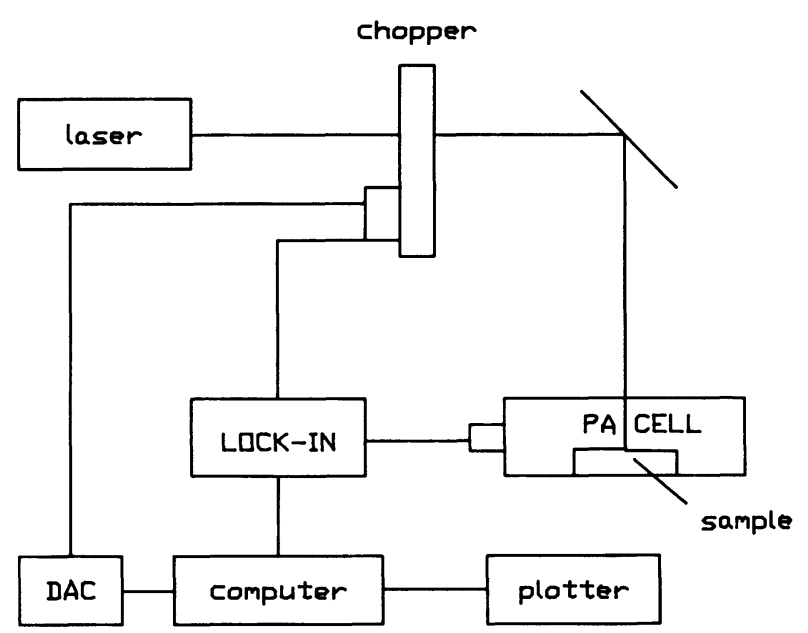

Fig. 1. - Schematic diagram of the experimental setup.

The Bennett and Patty formula [4] for the surface temperature of highly absorbing thin samples was used for initial analysis of the results :

$$
\begin{gathered}
S(\omega)=\frac{1+R_{\mathrm{b}} \exp \left(-2 q_{\mathrm{s}} \ell\right)}{1-R_{\mathrm{b}} R_{\mathrm{g}} \exp \left(-2 q_{\mathrm{s}} \ell\right)} \\
b=\sqrt{\frac{\rho_{\mathrm{b}} k_{\mathrm{b}} C_{\mathrm{b}}}{\rho_{\mathrm{s}} k_{\mathrm{s}} C_{\mathrm{s}}}}, \quad g=\sqrt{\frac{\rho_{\mathrm{g}} k_{\mathrm{g}} C_{\mathrm{g}}}{\rho_{\mathrm{s}} k_{\mathrm{s}} C_{\mathrm{s}}}}, \\
R_{\mathrm{b}}=(1-b) /(1+b), \quad R_{\mathrm{g}}=(1-g) /(1+g)
\end{gathered}
$$

where $S(\omega)$ is the complex ratio of the PA signals of thermally thin and thick samples from the same material, $\ell$ being the thickness of the former.

$$
q_{s}=(1+i) \sqrt{\omega \rho_{\mathrm{s}} C_{\mathrm{s}} / 2 k_{\mathrm{s}}}=(1+i) / \mu_{\mathrm{s}}
$$

is the complex thermal wave number; $i$ is the imaginary unit; $\omega$ is the angular modulation fre- quency; $\rho, k$, and $C$ are the density, the thermal conductivity, and the specific heat of the coating (s), the backing (b), and the surrounding gas (g) respectively ; $\mu_{s}$ is the coating thermal diffusion length. In our calculations we assume $R_{\mathrm{g}}=1$.

The results of the investigations of S1 and S2 are presented in figure 2 and figure 3 along with the least square fit of the theoretical curves (1). S3 was used as a reference (thermally thick) sample in this case because it was made from the same powder and was sprayed under the same conditions. For the same reasons the reference samples for S5 and S11 were S6 and S12 respectively. Since the backing parameters are known the thermal conductivity and the specific heat of these coatings can be derived.

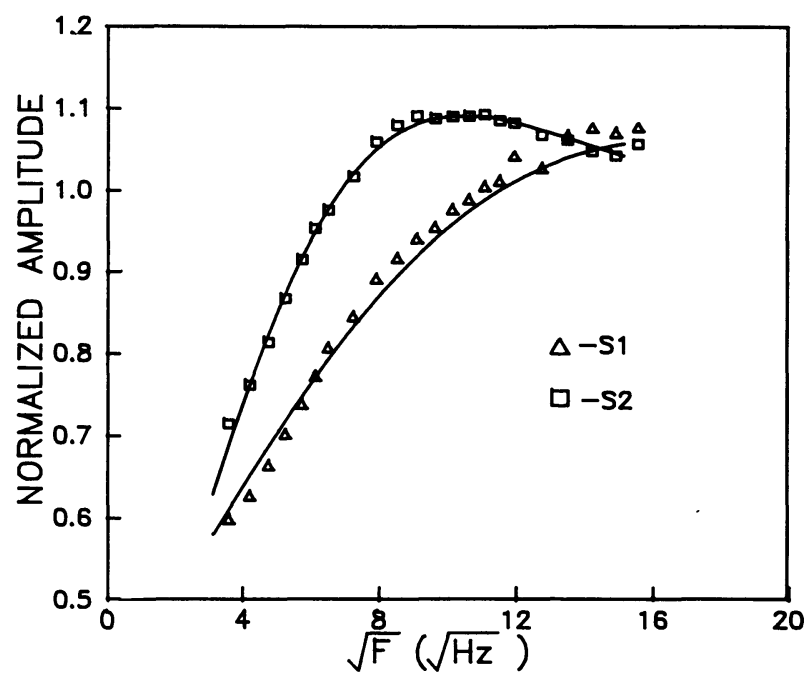

Fig. 2. - Normalized amplitude of $S 1(\triangle)$ and $S 2(\square)$ along with the corresponding theoretical curves (1) vs. the square root of the frequency.

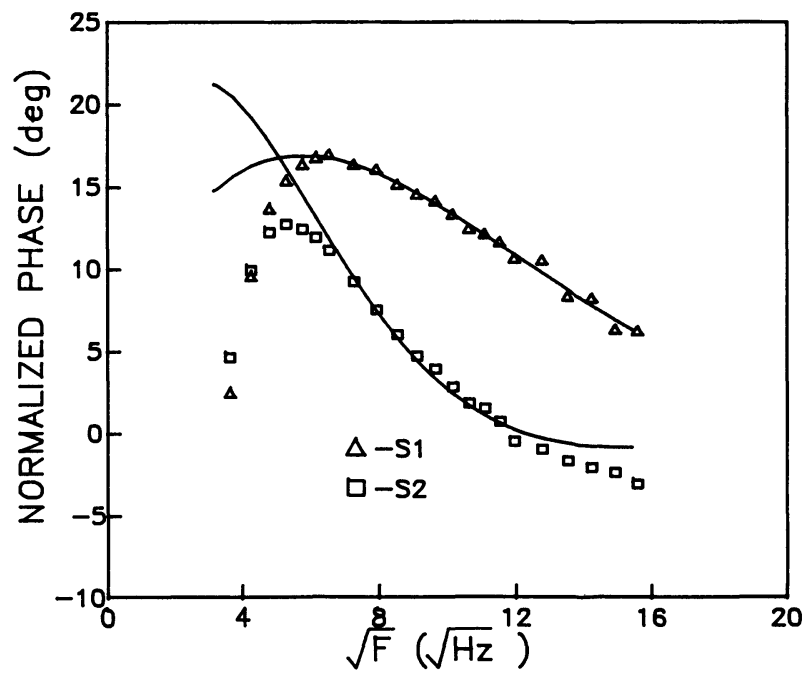

Fig. 3. - Normalized phase of $S 1(\triangle)$ and $S 2(\square)$ along with the corresponding theoretical curves (1) vs. the square root of the frequency. 
Data obtained from phase and amplitude measurements for different samples are given in table II.

Table II. - Results from amplitude and phase analyses.

\begin{tabular}{|l|c|c|c|c|}
\hline Sample & \multicolumn{2}{|c|}{$k_{\mathrm{s}}(\mathrm{W} / \mathrm{m}-\mathrm{K})$} & \multicolumn{2}{c|}{$C_{\mathrm{s}}(\mathrm{J} / \mathrm{kg}-\mathrm{K})$} \\
\hline & amplitude & phase & amplitude & phase \\
\hline S1 & 4.45 & 4.67 & 753 & 718 \\
S2 & 3.1 & 4.6 & 500 & 654 \\
S5 & 3.61 & 4.12 & 534 & 657 \\
S11 & 37 & 34 & 510 & 492 \\
\hline
\end{tabular}

It is seen that below $50 \mathrm{~Hz}$ the Bennett and Patty formula (1) can not describe the PA behaviour of S1 and $\mathbf{S} 2$, while the experimental results from the sample S5 (Fig. 4 and Fig. 5), sprayed from the same powder but under different conditions, agree very well with this formula. Most likely this difference is due to changes of the coating structure. Some of the possible reasons for the changes may be :

1) at these frequencies the reference sample $S 3$ itself becomes thermally thin;

2) presence of pores and inclusions ;

3) presence of thermal barriers between the particles.

In the first case one can find out from the obtained thermal parameters that even at $10 \mathrm{~Hz}$ the thermal diffusion length of the coating is more than 10 times smaller than its thickness.

In order to determine more precisely the reason for this difference the photoacoustic phases of S3,

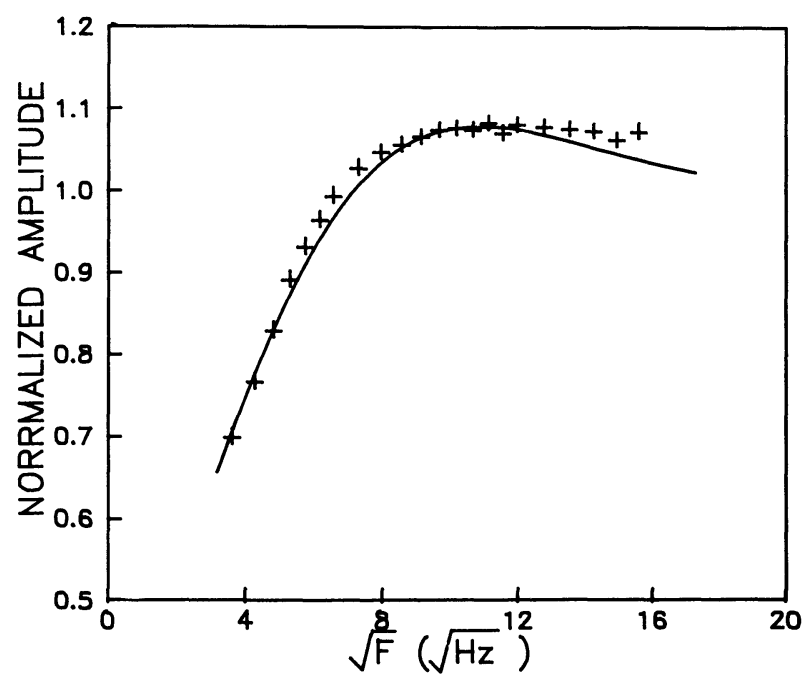

Fig. 4. - Normalized amplitude of S5 along with the corresponding theoretical curve (1) vs. the square root of the frequency.

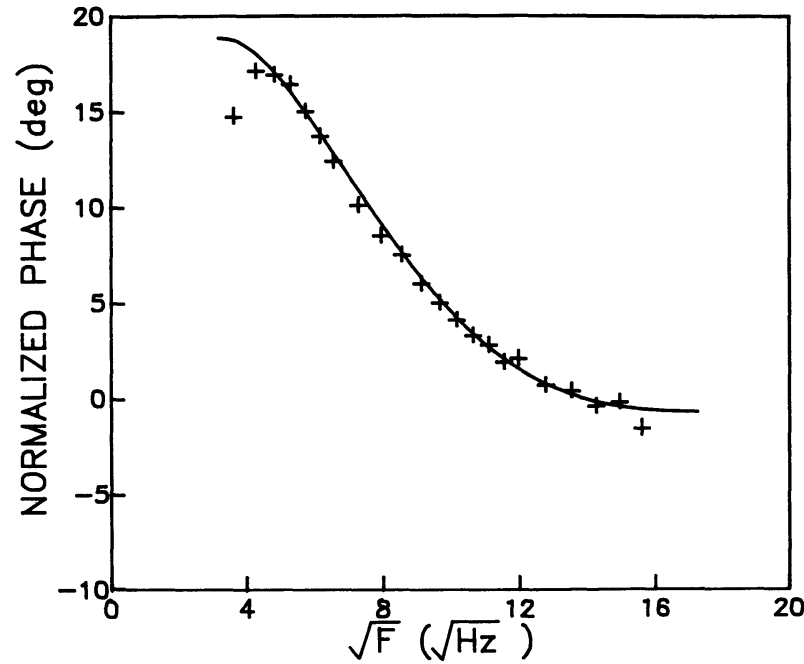

Fig. 5. - Normalized phase of S5 along with the corresponding theoretical curve (1) vs. the square root of the frequency.

S6, S9, and S12 were compared to the one from a blackened optical glass and the results are shown in figure 6. From Rosencwaig-Gersho theory [5] it follows that the phase shift between two opaque, thermally thick samples is 0 . This holds true to a great extent for S6, but the relative phase of S3 changes rapidly below $50 \mathrm{~Hz}$, where (1) can not explain the experimental data (Fig. 3). The phase shifts of the other two samples also differ from the theory of homogeneous media.

The PA signal from porous and powdered samples has been discussed by several authors [6-8]. It has been shown that the gas phase influence in such

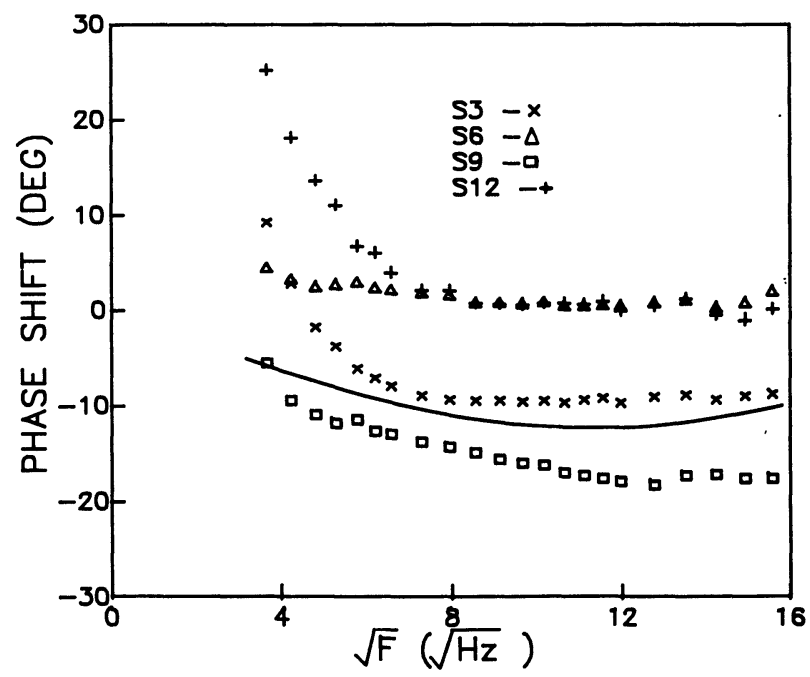

Fig. 6. - Phase shift between the thick coatings and a blacken optical glass $(S 3(\times), S 6(\triangle), S 9(\square)$, and $S 12(+))$. The solid line is the normalized phase of the surface temperature according to the Opsal-Rosencwaig theory [8]. 
samples is twofold. On the one hand it changes the sample surface temperature whose periodic variations give rise to the expansion and the contraction of the gas above the sample. On the other hand the expansion of the interstitial gas can enhance significantly the PA signal. In other words the composite piston model $[6,7]$ have to be used instead of the thermal piston model [5]. The influence of the pores on the sample temperature is taken into account by introducing an effective thermal conductive parameter (see Eq. (11) and Appendix A in [6] and Eq. (15) in [8]), which is derived for the limit of steady-state temperature distribution. In fact this thermal conductive parameter depends on the modulation frequency and not considering this dependence means that thermal wave scattering and reflection are neglected.

Since the pores in sprayed coatings don't form continuous phase the "pressure " term in the composite piston model can be neglected and RosencwaigGersho theory can be used. Recently a theory for the temperature distribution in porous medium has been developed and experimentally proven [9]. An uniform light flux illuminates highly absorbing semiinfinite porous space. Vacuum pores in the form of parallelepipeds are situated in uniform layers (Fig. 7a). Because of the symmetry the heat diffusion process may be examined only within $1 / 8$ part of the square rod marked off in figure $7 \mathrm{a}$. In figure $7 \mathrm{~b}$ the magnified prismatic domain where the heat equation is to be solved is shown. It is obvious that the average surface temperature obtained in this way is equal to that of the whole surface. In the solid phase the thermal conductivity equation holds. In the case of sinusoidally modulated source it can be written as follows :

$$
\Delta T-q^{2} T=0 .
$$

The following boundary conditions are to be satisfied :

(i) $-\left.k \frac{\partial T}{\partial z}\right|_{z=0}=I \exp (i \omega t), I$ is the intensity of the chopped light,

(ii) absence of heat flow between the solid phase and pores,

(iii) absence of heat flow through the lateral surface, because of the symmetry.

Under these conditions the final formula for the average surface temperature $\langle T\rangle_{z=0}$ is obtained as follows :

$$
\begin{aligned}
& \langle T\rangle_{z=0}=\frac{I}{k q} \times \\
& \times\left[1+\frac{4 \alpha \phi}{\alpha h-f+\sqrt{(\alpha h-f)^{2}+4 g \phi \alpha}-2 \alpha \phi}\right]
\end{aligned}
$$

$$
\begin{aligned}
f= & \left(1-\frac{(\gamma p)^{2 / 3}}{2}\right)^{2}-\left(\frac{(\gamma p)^{2 / 3}}{2}\right)^{2} \times \\
& \times \exp \left[2 q d\left(p / \gamma^{2}\right)^{1 / 3}\right] \\
h= & \left(1-\frac{(\gamma p)^{2 / 3}}{2}\right)^{2}-\left(\frac{(\gamma p)^{2 / 3}}{2}\right)^{2} \times \\
& \times \exp \left[-2 q d\left(p / \gamma^{2}\right)^{1 / 3}\right] \\
g= & \left(1-\frac{(\gamma p)^{2 / 3}}{2}\right)\left(\frac{(\gamma p)^{2 / 3}}{2}\right) \times \\
& \times\left(1-\exp \left[2 q d\left(p / \gamma^{2}\right)^{1 / 3}\right]\right) \exp \left(2 q z_{1}\right) \\
\phi= & \left(1-\frac{(\gamma p)^{2 / 3}}{2}\right)\left(\frac{(\gamma p)^{2 / 3}}{2}\right) \times \\
& \times\left(1-\exp \left[-2 q d\left(p / \gamma^{2}\right)^{1 / 3}\right]\right) \exp \left(-2 q z_{1}\right) \\
& \quad \alpha=\exp (2 q d), a / b=\gamma c / d
\end{aligned}
$$

where $p$ is the porosity, and $\gamma$ is a coefficient introduced to take into account the form of the pores. In figure 8 the phase difference between a porous sample with different values of $\gamma$ and a homogeneous one is plotted $\left(z_{1}=(d-c) / 2\right)$. The thermal parameters and the porosity were taken from the measurements. The average size and the approximate form of the pores $(\gamma>1)$ where obtained from micrographs of the cross sections of S3 and S6 (Fig. 9). At the frequencies used in the experiments the phase shift is less than $3^{\circ}$ and its maximum is about $1 \mathrm{kHz}$ where the thermal wave length becomes comparable to the size of the pores and the diffraction is considerable. It is obvious that in this case pores with the observed form and distribution have little influence on the photoacoustic signal and moreover the porosity of S3 and S6 is almost the same.

A problem not tackled here is how " open " pores and surface roughness affect the PA signal. The reason is that S3 and S6 have the same surface structure and evidently it doesn't cause the phase difference between the two samples. On the other hand the thermal diffusion length of these coatings $(\sim 50 \mu \mathrm{m}$ at $250 \mathrm{~Hz})$ is greater than the characteristic depth of the surface roughness [8], which decreases the surface structure influence.

During the projection of the particles towards the substrate the temperature rises and the grains are partly melted which contributes to their fusion to one another. At lower temperatures the fusion is not sufficient and thermal barriers between the grains arise (the presence of thermal barriers in ceramics was experimentally proved in [10]). The particles in these coatings have an elongated form. If the fusion is insufficient the sprayed coatings might be modelled by layers of good thermal conductivity separated by thermal barriers (Fig. 10). Samples with alternating layers of high and low thermal conductivity are investigated in [1] but only the influence of the first poor thermal conductive layer is analysed. A conclusion has been drawn that if the layer is thin, it 


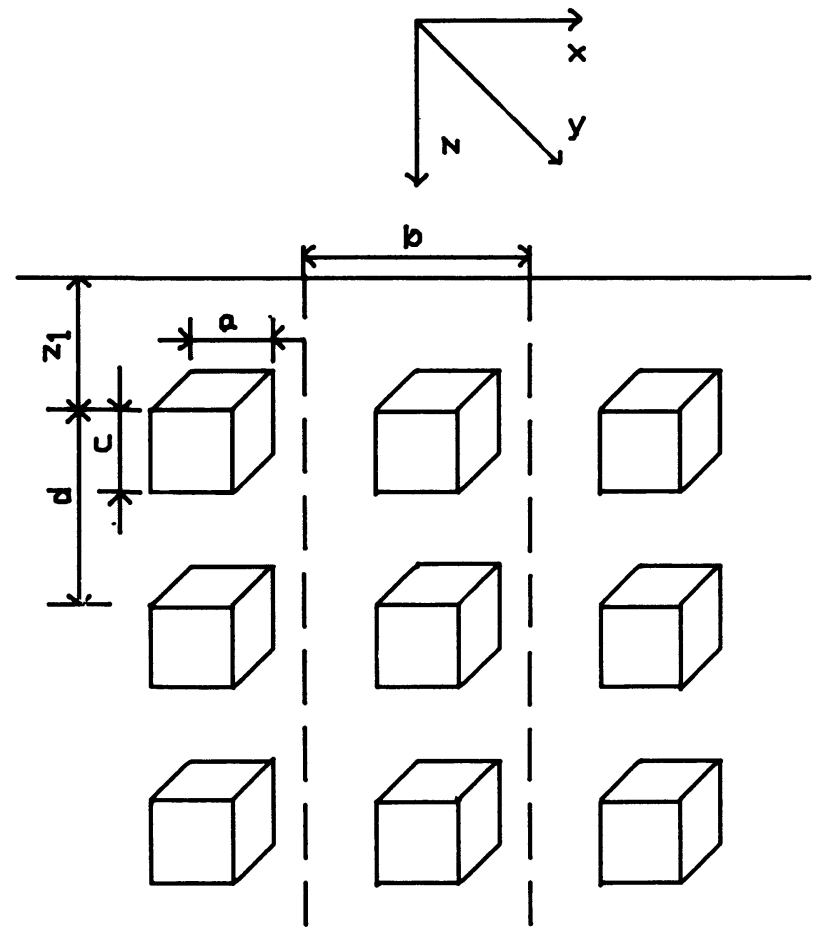

a)
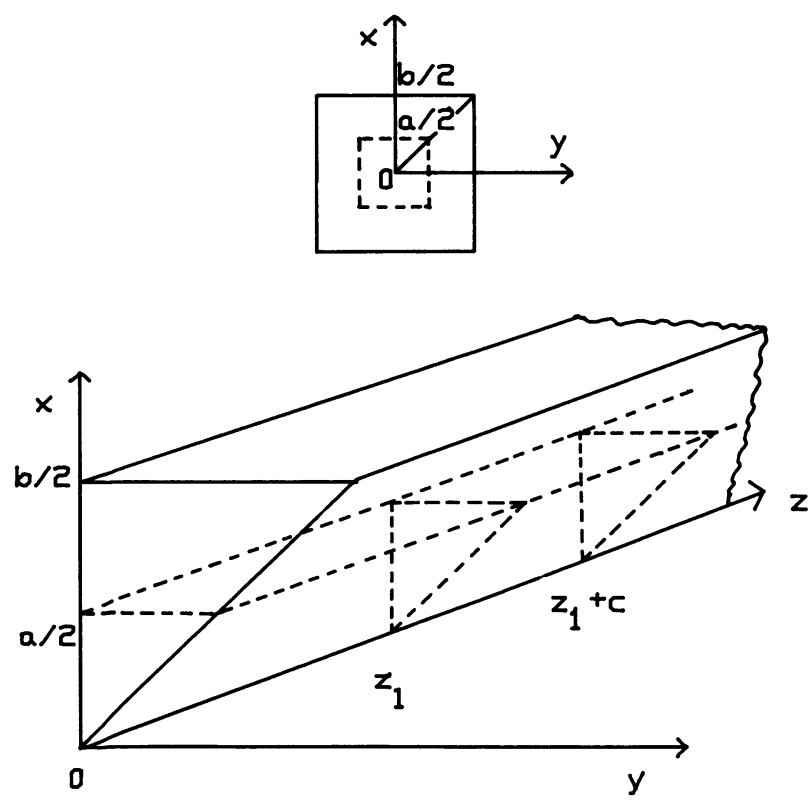

b)

Fig. 7. - (a) Model of porous medium; (b) Domain where the heat equation is solved.

doesn't affect the thermal parameters of the coating. The taking into account of only the first three layers corresponds to high frequency of modulation and strong attenuation of the thermal waves. It is seen from figure 6 that at frequencies above $50 \mathrm{~Hz}$ the phase shifts of the samples are constant (the phase of S9 slightly changes). At lower frequencies the ther-

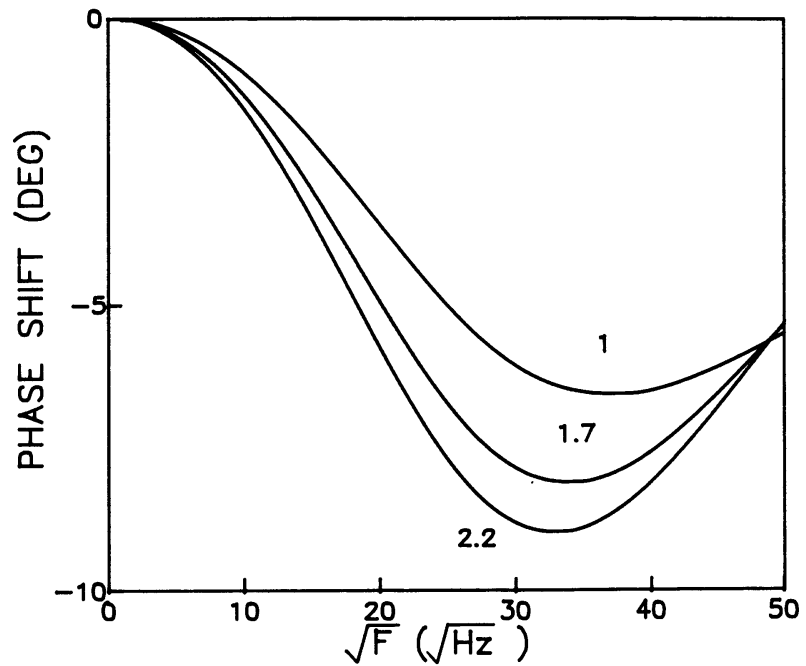

Fig. 8. - Phase lag of a porous sample vs. the square root of the frequency. The porosity is 0.17 , and the curves are labeled with $\gamma$.

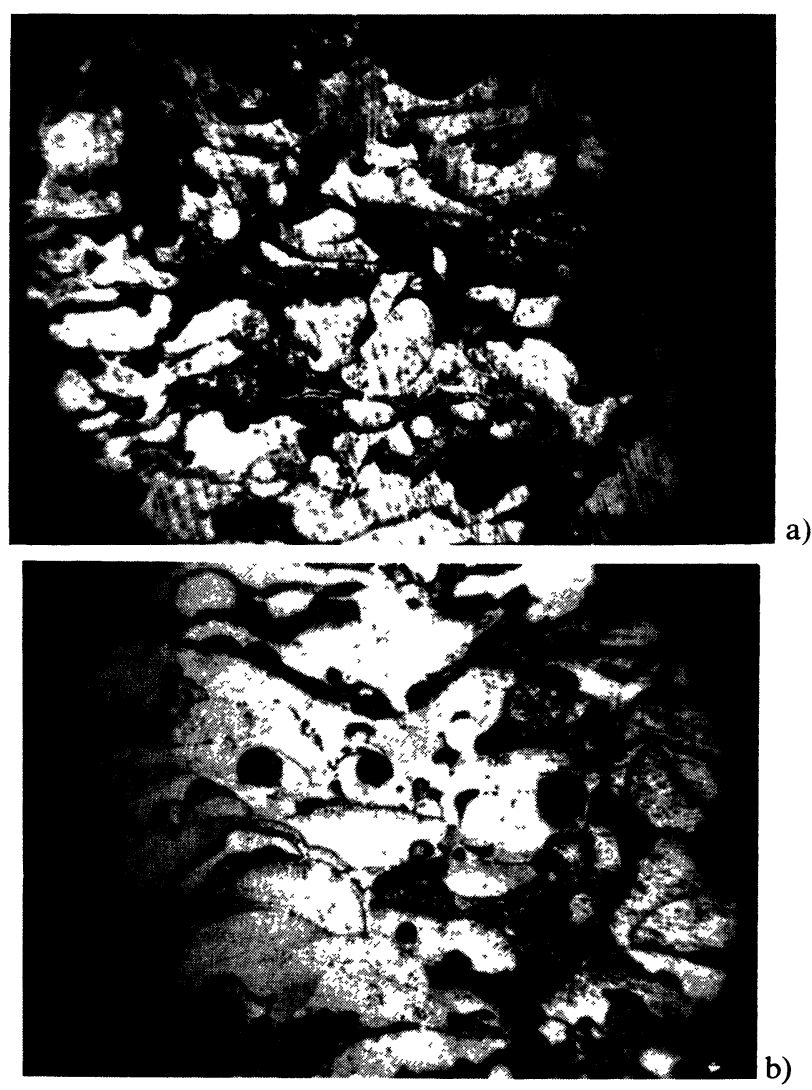

Fig. 9. - Micrographs of the cross sections of S3 (a) and S6 (b).

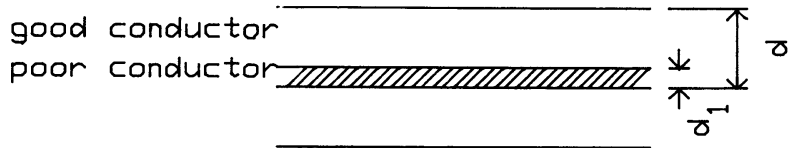

IIIm

Fig. 10. - Layered model of thermally sprayed coating. 
mal waves cover several layers with low thermal conductivity and they have to be taken into account. The thermal behaviour of layered systems is described by the Opsal-Rosencwaig theory [11]. The relative phase of a layered sample with realistic parameters (mean particle size $d=35 \mu \mathrm{m}$, $d_{1}=0.2 d$, and a ratio between the thermal conductivities $1 / 10$ ) is shown in figure 6 with solid line. It is seen that the theoretical curve is in agreement with the experimental data.

Thus the comparison of the phases from the thick samples S3, S6, S9, S12 and the phase from a blackened optical glass allows some of the coatings to be modelled by layered systems. Probably the use of air to additionally accelerate the projected grains deteriorates the thermal contact between them and such coatings can not be considered thermally homogeneous. This explains why it is impossible to fit the low frequency data from S1 and S2 with the theoretical curves (1).

\section{Conclusion.}

It has been experimentally proven that under certain conditions the "average" thermal parameters of highly heterogeneous coatings can be determined. It is seen from the micrographs that besides porosity there are three different phases in the coatings. While on the micrographs of the cross sections it was difficult to observe any difference between samples from the same powder, sprayed under different conditions, the thermal wave investigation showed an obvious distinction in their structure. A theoretical study of the influence of pores and thermal barriers was conducted in order to interpret the photoacoustic behaviour of some samples and the thermal barriers were found to affect significantly the coating parameters. It is to noting that this problem is too complex and different techniques have to be applied in order to establish the relationship between the spraying conditions, the microstructures and the thermal properties of sprayed coatings.

References

[1] Patel P. M., Almond D. P., J. Mater. Sci. 20 (1985) 955.

[2] Cielo P., J. Appl. Phys. 56 (1984) 230.

[3] Morris J., Patel P. M., Almond D. P., Reiter H., Surf. Coat. Techn. 34 (1988) 51.

[4] Bennett C. A. Jr, Patty R. R., Appl. Opt. 21 (1982) 49.

[5] Rosencwaig A., Gersho A., J. Appl. Phys. 47 (1976) 64

[6] Monchalin J.-P., Bertrand L., Rousset G., Lepoutre F., J. Appl. Phys. 56 (1984) 190.
[7] McGovern S., Royce B., Benziger J., J. Appl. Phys. 57 (1985) 1710.

[8] Bein B., Krueger S., Pelzl J., Can. J. Phys. 64 (1986) 1208.

[9] Velinov Ts., Gusev V., Bransalov Kl., submitted to Electron. Lett.

[10] Lepoutre F., Legal Lasalle E., Sachet J., BOCCARA A., 6th International Topical Meeting on Photoacoustic and Photothermal Phenomena July 31 - August 3 1989, Baltimore, 82.

[11] Opsal J., Rosencwaig A., J. Appl. Phys. 53 (1982) 4240. 